\title{
Políticas de distribuição com lote econômico de entrega em problemas de roteirização com estoque gerenciado pelo fornecedor e sistema logístico em três níveis
}

\section{Economic order quantity distribution policies in vendor-managed Inventory Routing Problems and Three-level Distribution System}

\author{
Thiago Guimarães ${ }^{1,2}$ \\ Cassius Scarpin ${ }^{3}$ \\ Maria Teresinha Steiner ${ }^{4}$
}

\begin{abstract}
Resumo: Em sistemas de gerenciamento de estoque pelo fornecedor (Vendor Managed Inventory - VMI), as decisões logísticas são centralizadas no Centro de Distribuição (CD), possibilitando uma redução simultânea dos custos de armazenagem e transporte. Sua operação requer a resolução de um complexo problema de otimização combinatória, denominado Problema de Roteirização e Estoques (PRE) que consiste no gerenciamento do estoque do cliente, no estabelecimento da frequência e quantidade de produto entregue, além do roteiro percorrido pelo veículo ao longo de um horizonte de planejamento. Este trabalho apresenta três novas políticas de distribuição baseadas no cálculo de lote econômico de entrega para o PRE. Um modelo de Programação Linear Inteira Mista Binária (PLIMB) foi proposto considerando um sistema logístico de três níveis, sendo uma Base de Fornecimento (BF), um CD e um conjunto de clientes. Também foi proposta uma nova abordagem heurística que decompõe o PRE em dois subproblemas: o primeiro é responsável pela programação das entregas e a formação de grupos de atendimento ao longo do horizonte de planejamento. O segundo subproblema constrói os roteiros para os grupos formados no primeiro estágio, empregando heurísticas clássicas de construção de rotas, juntamente com procedimento de melhorias intrarrotas. Ajustes entre rotas são realizados por uma nova estratégia de busca ampla em vizinhança. Cenários de pequeno, médio e grande porte, com variações no custo de estocagem e de transporte, foram gerados a partir de dados da literatura e parametrizados no contexto da pesquisa. Extensivos testes computacionais foram conduzidos a fim de evidenciar a eficiência das políticas de distribuição propostas e a efetividade da estratégia heurística utilizada.

Palavras-chave: Lote Econômico de Entrega. Roteirização e Estoques. Sistemas Logísticos de Três Elos. Heurísticas de Decomposição.
\end{abstract}

\begin{abstract}
In vendor-managed inventory systems, logistics decisions are centralized at the vendor, allowing inventory storage and transportation costs to be reduced simultaneously. The operation of such systems requires the solution of a complex combinatorial optimization problem, known as Inventory Routing Problem (IRP), which involves managing client inventory and determining the frequency and size of product deliveries as well as the route taken by the vehicle over a given planning horizon. We present three new dynamic formulations for an economic order quantity distribution policy for the multivehicle inventory routing problem (MIRP). A mixed-integer linear programming (MILP) model with additional practical constraints was used for the MIRP, considering a three-echelon logistic system. A new heuristic approach that breaks the MIRP down into the following two sub-problems was also proposed: the first sub-problem deals with the scheduling of deliveries and the formation of delivery clusters over the planning horizon; the second sub-problem builds the routes for the delivery clusters using classic route construction heuristics and a procedure for intra-route improvements. Adjustments between routes are performed with the aid of a new large neighborhood search (LNS) strategy. Small, medium, and large scenarios with different storage and transportation costs were generated using parameters based on data from the literature. Extensive computational tests were carried out to determine the effectiveness of the proposed distribution policy and the heuristic used.
\end{abstract}

Keywords: Economic Order Quantity Policy. Inventory Routing. Three-echelon Logistic System. Decomposition Heuristic Approach.

\footnotetext{
Centro Universitário Franciscano do Paraná - UNIFAE, CEP 80230-080, Curitiba, PR, Brasil

2 Programa de Pós-graduação em Métodos Numéricos em Engenharia, Universidade Federal do Paraná - UFPR, CP 19081, CEP 81531-990, Curitiba, PR, Brasil, e-mail: thiandgui@gmail.com

3 Programa de Pós-graduação em Métodos Numéricos em Engenharia e Programa de Pós-graduação em Engenharia de Produção, Universidade Federal do Paraná - UFPR, CP 19081, CEP 81531-990, Curitiba, PR, Brasil, e-mail: cassiusts@ gmail.com

${ }^{4}$ Programa de Pós-graduação em Engenharia de Produção e Sistemas, Pontifícia Universidade Católica do Paraná - PUCPR, Rua Imaculada Conceição, 1155, Prado Velho, CEP 80215-901, Curitiba, PR, Brasil, e-mail: maria.steiner@pucpr.br
}

Recebido em Out. 23, 2013 - Aceito em Ago. 5, 2014

Suporte financeiro: CAPES e CNpQ. 


\section{Introdução}

Sistemas VMI são amplamente reconhecidos por criarem valor tanto para o fornecedor quanto para o cliente, em uma situação de ganha-ganha. $\mathrm{O}$ fornecedor beneficia-se pela maior acurácia no planejamento da produção e distribuição de seus produtos, possibilitando-o reduzir os níveis de ruptura e elevar o grau de coordenação das entregas. Já o cliente elimina a necessidade de gerenciar seus estoques, deixando de incorrer nos custos associados a este processo (ÖZENER; ERGUN; SAVELSBERGH, 2013). Todavia, a operação otimizada de sistemas $V M I$ implica a resolução de um complexo problema de otimização combinatória denominado Problema de Roteirização e Estoques (PRE). O PRE combina em um mesmo contexto o gerenciamento de estoque e as decisões de roteirização de veículos ao longo de um horizonte de planejamento (COELHO et al., 2012c).

Por sua relevância prática e grande contribuição ao gerenciamento integrado de sistemas de produção e distribuição, o PRE vem recebendo ampla atenção da literatura acadêmica nos últimos 30 anos (COELHO; CORDEAU; LAPORTE, 2013). É possível destacar inúmeras aplicações do PRE, como na distribuição de produtos derivados de petróleo por meio marítimo (GRONHAUG et al., 2010; RAKKE et al., 2011); em problemas de distribuição e armazenagem de combustíveis utilizando veículos terrestres compartimentados (POPOVIĆ; VIDOVIĆ; RADIVOJEVIĆ, 2012); na entrega de produtos perecíveis (GUMASTA; CHAN; TIWARI, 2012; HIASSAT; DIABAT, 2010); na distribuição de sangue e plasma para hospitais (HEMMELMAYR et al., 2010); na logística de distribuição de cimento a granel entre fábricas e silos por meio marítimo com frota heterogênea (CHRISTIANSEN et al., 2011); na logística de componentes da indústria automotiva (ALEGRE; LAGUNA; PACHECO, 2007); entre outras.

Em seus aspectos teóricos, o PRE pode ser pormenorizado conforme alguns critérios de classificação, envolvendo: horizonte de tempo (finito ou infinito); composição da frota (homogênea ou heterogênea); demanda dos clientes (determinística ou estocástica); construção de roteiros (direta, múltipla ou contínua); e política de reposição. Outrossim, este último critério define as regras principais sobre a quantidade entregue aos clientes, impactando diretamente o custo total. Neste contexto, a política maximum level $(M L)$ flexibiliza a quantidade entregue ao cliente, estando limitada à sua disponibilidade de armazenamento no período, enquanto a política order-up-to Level (OU) impõe ao fornecedor entregar a máxima quantidade possível ao cliente, sempre que este for visitado. Geralmente, a política $M L$ tende a prevalecer sobre a política $O U$ (COELHO et al., 2012c; COELHO, 2012; ARCHETTI et al., 2011) na medida em que a quantidade entregue pode ser determinada de maneira pontual para cada cliente de acordo com as características de momento do problema. Todavia, uma formulação $M L$ mais direcionada à minimização dos estoques pode ocasionar um tradeoff entre os custos de armazenagem e os custos de transporte entre tais políticas.

Diante do exposto, este estudo tem por objetivo encontrar um ponto de equilíbrio entre as decisões de transporte e estocagem capaz de minimizar o custo total de operação de sistemas VMI. Para tal, o artigo apresenta três novas formulações de políticas de distribuiçãa para o PRE por meio do cálculo dinâmico de Lotes Econômicos de Entrega (LE). Ademais, o trabalho incorpora um sistema logístico em três níveis, no qual o Centro de Distribuição (CD) pode decidir em que período receberá uma quantidade fixa de produto oriundo de uma Base de Fornecimento (BF). Esta generalização, introduzida por Zhao, Chen e Zang (2008), é escassamente tratada em outros trabalhos e ainda não se reporta na literatura uma abordagem que considere políticas de distribuição do tipo LE em tal estrutura.

A motivação prática do estudo refere-se à otimização da logística de distribuição de Gás Liquefeito de Petróleo (GLP) a granel em ambientes urbanos. Em linhas gerais, uma empresa deste segmento coordena suas decisões logísticas a partir de um CD e precisa planejar o reabastecimento de seu estoque a partir de uma BF, além de programar entregas periódicas para um conjunto de clientes dispersos geograficamente. A aplicação de sistemas VMI neste tipo de situação pode constituir grande diferencial competitivo.

O restante do artigo está estruturado como segue: na seção 2, o PRE é formalmente definido como um modelo PLIMB, de acordo com as políticas de distribuição $M L$ e $O U$. A seção 3 apresenta as formulações da política de distribuição por lote econômico. A seção 4 detalha a heurística desenvolvida. Na seção 5, os cenários gerados e os resultados obtidos são explanados. A seção 6 conclui o estudo.

\section{Formalização do problema}

O Problema de Roteirização e de Estoques é formulado sobre um grafo completo e não orientado $G=(V, A)$ em que $V=\{0, \ldots, n\}$ é o conjunto de vértices e $A=\{(i, j) \in V, i \neq j\}$, o conjunto de arcos. O vértice " 0 " refere-se ao depósito e $V$ ' $=V \backslash\{0\}$ representa os clientes. Cada arco $(i, j) \in V$ possui um custo não negativo $c_{\mathrm{ij}}$ e a distância entre o vértice $0(\mathrm{CD}) \mathrm{e}$ a BF é $\xi$. As decisões são definidas em horizonte $T=\{0, \ldots, p\}$. O cliente $i$ demanda $d_{i}^{t}, \forall t \in T$, possui um custo de estocagem $h_{\mathrm{i}}$ e uma capacidade de 
armazenamento $C_{\mathrm{i}}$, sendo $\min C_{\mathrm{i}}$ a quantidade mínima que deve ser mantida no estoque, calculada como uma proporção de $C_{\mathrm{i}}$. No instante $t=0$, é conhecido o estoque inicial $I_{i}^{0}, \forall i \in V$. Assume-se que a BF possui uma quantidade suficiente de produto para atender à demanda dos clientes ao longo de $p, \mathrm{e}$ o CD deve escolher em que período $t$ será abastecido com uma carga fixa $r$ a partir de BF.

Uma frota homogênea de $K$ veículos, $k=\{1, \ldots K\}$, com capacidade $Q$ está disponível no CD. Cada veículo $k$ realiza uma única rota em cada período $t$, limitado a um número máximo de clientes atendidos $N$. Conforme demonstrado na Figura 1, o processo de distribuição envolve duas etapas: a primeira é da $\mathrm{BF}$ para o $\mathrm{CD}$ e a segunda etapa parte do $\mathrm{CD}$ para um conjunto de clientes $(\mathrm{V})$.

As variáveis de decisão são descritas como:

- $x_{i j k}^{t}=1$ se o arco $(i, j)$ é percorrido no período $t$ pelo veículo $k$ e " 0 ", caso contrário;

- $\quad y_{i k}^{t}=1$ se o cliente $i$ é visitado pelo veículo $k$ no período $t$ e " 0 ", caso contrário;

- $\lambda^{t}=1$ se o CD é recarregado com $r$ no período $t$ e " 0 ", caso contrário;

- $I_{i}^{t}$ : nível de estoque do cliente $i$ ao término do período $t$

- $I_{0}^{t}$ : nível de estoque do depósito ao término do período $t$;

- $q_{i k}^{t}$ : quantidade entregue ao cliente $i$ no período $t$ pelo veículo $k$;

- $f_{i j}^{t}$ : fluxo de veículos que chega ao nó $j$ a partir do nó $i$ no período $t$.

O objetivo do PRE é minimizar o custo total de distribuição e armazenamento, endereçado à política do $V M I$, atendendo às seguintes restrições adicionais:

- O estoque $I_{i}^{t}$ do cliente $i$ não deve exceder sua capacidade $C_{\mathrm{i}}$ nem ser inferior ao mínimo requerido $\min C_{\mathrm{i}}$ ao longo de $p$.

- O estoque $I_{0}^{t}$ do depósito não pode ser negativo ao longo de $p$.

- Cada cliente $i$ deve ser atendido por um único veículo.

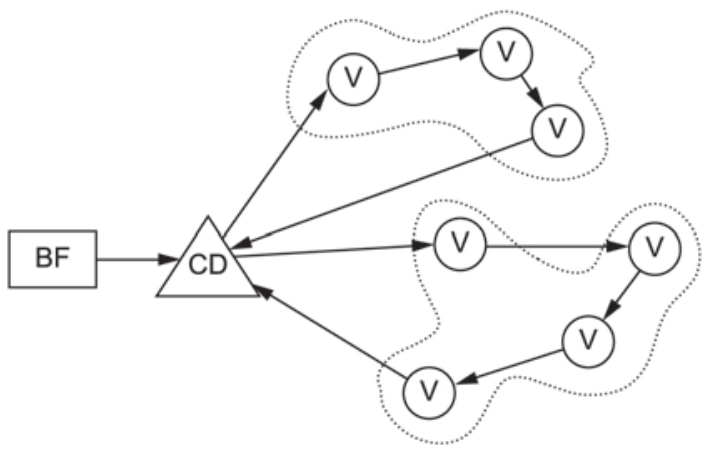

Figura 1. Sistema logístico em três níveis. Fonte: Adaptado de Zhao, Chen e Zang (2008).
- A totalidade entregue pelo veículo $k$ no período $t$ não deve exceder sua capacidade $Q$.

- Cada veículo deve realizar apenas uma rota de entrega por período, devendo esta iniciar e terminar no depósito e a quantidade de rotas não deve exceder a disponibilidade da frota.

Baseado em Coelho, Cordeau e Laporte (2012a), o PRE em um sistema logístico de três níveis e política $M L$ pode ser formulado como um modelo de PLIMB como segue:

Minimizar

$$
\sum_{t=1}^{p} I_{0}^{t} h_{0}+\sum_{t=1}^{p} \lambda^{t} \xi+\sum_{i=1}^{n} \sum_{t=1}^{p} I_{i}^{t} h_{i}+\sum_{i=0}^{n} \sum_{j=0, j \neq i}^{n} \sum_{k=1}^{K} \sum_{t=1}^{p} c_{i j} x_{i j k}^{t}
$$

Sujeito à:

$$
\begin{aligned}
& I_{i}^{t} \leq C_{i} \quad \forall t \in T, i \in V^{\prime} \\
& I_{i}^{t} \geq \min C_{i} \quad \forall t \in T, i \in V^{\prime} \\
& \sum_{k=1}^{K} q_{i k}^{t} \geq d_{i}^{t}-\left(I_{i}^{t-1}-\min C_{i}\right) \quad t \in T, i \in V^{\prime} \\
& q_{i k}^{t} \leq C_{i} y_{i k}^{t} \quad \forall k \in K, t \in T, i \in V^{\prime} \\
& I_{i}^{t}=I_{i}^{t-1}+\sum_{k=1}^{K} q_{i k}^{t}-d_{i}^{t} \quad \forall t \in T, i \in V^{\prime} \\
& I_{0}^{t}=I_{0}^{t-1}+r \lambda^{t}-\sum_{i=1}^{n} \sum_{k=1}^{K} q_{i k}^{t} \quad \forall t \in T \\
& \sum_{i=0}^{n} q_{i k}^{t} \leq Q \quad \forall k \in K, t \in T \\
& \sum_{i=1}^{n} y_{i k}^{t} \leq N \quad \forall k \in K, t \in T \\
& \sum_{k=1}^{K} y_{i k}^{t} \leq 1 \quad \forall i \in V^{\prime}, t \in T \\
& \sum_{i=1}^{n} x_{i j k}^{t}=\sum_{i=1}^{n} x_{j i k}^{t}=1 \quad \forall k \in K, t \in T, j \in V^{\prime} \\
& \sum_{i=1}^{n} x_{0 i k}^{t}=\sum_{i=1}^{n} x_{i 0 k}^{t} \quad \forall k \in K, t \in T \\
& \sum_{k=1}^{K} y_{0 k}^{t} \leq K \quad \forall t \in T \\
& \sum_{i=0}^{n} x_{i j k}^{t}+\sum_{i=0}^{n} x_{j i k}^{t}=2 y_{i k}^{t} \quad \forall k \in K, t \in T \\
& \sum_{j=0}^{n} \sum_{k=1}^{K} f_{j i k}^{t}-\sum_{j=0}^{n} \sum_{k=1}^{K} f_{i j k}^{t}=\sum_{k=1}^{K} q_{i k}^{t} y_{i k}^{t} \quad \forall t \in T, i \in V^{\prime} \\
& f_{i j k}^{t} \leq Q y_{i k}^{t} \quad \forall k \in K, t \in T, i \in V, j \in V^{\prime} \\
& f_{i j k}^{t} \geq 0 \quad \forall k \in K, t \in T, i \in V, j \in V^{\prime} \\
& I_{i}^{t} \geq 0 \quad \forall t \in T, i \in V \\
& q_{i k}^{t} \geq 0 \quad \forall k \in K, t \in T, i \in V^{\prime} \\
& d_{i}^{t} \geq 0 \quad \forall t \in T, i \in V^{\prime}
\end{aligned}
$$




$$
x_{i j k}^{t}, y_{i k}^{t}, \lambda^{t} \in\{0,1\} \quad \forall k \in K, t \in T, i \in V, j \in V
$$

A expressão (1) minimiza a função objetivo do problema, contemplando o custo de armazenagem e abastecimento do depósito, o custo de armazenagem do cliente e o custo total de distribuição. O conjunto de inequações (2) e (3) limitam o estoque do cliente $i$. Em (4) e (5) garante-se que a quantidade entregue ao cliente $i$ no período $t$ atenda a sua necessidade e não exceda sua capacidade disponível. As equações em (6) e (7) calculam o estoque do cliente e do fornecedor, respectivamente. As inequações em (8) garantem que a quantidade transportada por um veículo $k$ não exceda sua capacidade, enquanto que o conjunto de inequações (9) limita a quantidade de clientes atendidos por um veículo a um parâmetro $N$ pré-determinado. Em (10), garante-se que cada cliente $i$ não seja visitado por mais de um veículo em $t$. Os conjuntos de restrições entre (11) e (15) modelam os requisitos para a não ocorrência de subciclos. O domínio das variáveis é definido entre (17) e (21).

\subsection{Política Order-Up-To Level}

A formulação da política $O U$ implica em adicionar restrições para a quantidade entregue, sendo abordada em Bertazzi, Paletta e Speranza (2005), Archetti et al. (2007) e Solyali e Sural (2011). Expandindo o modelo de Archetti et al. (2007) para múltiplos veículos, tem-se:

$$
\begin{aligned}
& q_{i k}^{t} \geq C_{i} y_{i k}^{t}-I_{i}^{t} \quad \forall k \in K, t \in T, i \in V^{\prime} \\
& q_{i k}^{t} \leq C_{i} y_{i k}^{t} \quad \forall k \in K, t \in T, i \in V^{\prime}
\end{aligned}
$$

As inequações asseguram ao cliente $i$ um carregamento igual à capacidade máxima de estocagem, dada por $C_{i}-I_{i}^{t}$ caso ele seja visitado pelo veículo $k$ no período $t\left(y_{i k}^{t}=1\right)$ e " 0 ", caso contrário $\left(y_{i k}^{t}=0\right)$.

Na medida em que a política $M L$ permite ao fornecedor realizar entregas em quantidades flexíveis dependendo do nível de estoque do cliente, estas tendem a ser mais frequentes, porém com abastecimentos em menor volume, reduzindo o custo de estocagem. Devido à menor quantidade entregue, o volume de estoque do fornecedor e o número de visitas aumentam, incrementando, portanto, o custo de estocagem no fornecedor e o custo de transporte. Já a política $O U$ eleva o nível de estoque dos clientes e consequentemente seu custo associado. Contudo, as visitas menos frequentes e a maior quantidade entregue reduzem o custo de estocagem do fornecedor e o custo de transporte. $\mathrm{O}$ maior volume entregue também implica maior número de recargas do $\mathrm{CD}$ a partir de $\mathrm{BF}$.

\subsection{Avanços recentes em Problemas de Roteirização e Estoques}

Pesquisas recentes acerca do PRE vêm incorporando restrições operacionais ao escopo teórico do problema, contudo não estão direcionados a investigar a questão da política de distribuição. A exceção do estudo de Coelho e Laporte (2013), que discute o impacto da adoção de níveis distintos de estoque no cliente, o que os autores denominaram de "política de nível ótimo" - optimized target level (OTL). O estudo aborda o PRE de forma exata por meio de uma técnica resolutiva via branch-and-cut. Já a programação das entregas é modelada como um problema de programação linear inteira binária e resolvida por um algoritmo de fluxo em redes. Entretanto, como já mencionado, a predominância das pesquisas está orientada a outras características do PRE.

Neste sentido, o trabalho de Liu e Lee (2011) introduz a restrição de janelas de tempo na entrega, considerando a política de distribuição $M L$. O trabalho de Coelho, Cordeau e Laporte (2012b), considera a possibilidade de transbordo do produto entre os clientes e trata das políticas $M L$ e $O U$. Já Coelho, Cordeau e Laporte (2012a), abordam o conceito de consistência em Problemas de Roteirização e Estoques. Neste trabalho, os autores avaliam os efeitos sobre o custo otimizado de operação de sistemas VMI de tratativas que visam elevar o nível de serviço, como a imposição de um intervalo mínimo e máximo para o cliente ser reabastecido, restrições que garantem que um mesmo veículo visite o cliente ao longo do período de planejamento, além do estabelecimento de limites mínimos de carregamento do veículo para se realizar uma entrega. A política $O U$ também é tratada como uma consistência do PRE. Já Coelho, Laporte e Cordeau (2012), expandem a possibilidade de transbordo para o caso estocástico do PRE, considerando as políticas $O U$ e $M L$. Mais recentemente Nishi e Izuno (2014), tratam da possibilidade de entregas fracionadas para um caso de PRE marítimo aplicado à distribuição de petróleo bruto. O Quadro 1 apresenta os avanços recentes sobre o tema.

\section{Políticas de distribuição com lote econômico de entrega}

Dado o trade-off entre custo de estocagem e custo de transporte nas políticas $M L$ e $O U$, o desenvolvimento de uma política de distribuição equilibrada é ensejado. Formulações para dimensionamento de lote econômico na distribuição de produtos podem ser encontradas em Sindhuchao et al. (2005), Birger e El-Houssaine (2008), Raa e Aghezzaf (2009) e Liu e Lee (2011). Com relação à gestão de estoques, Wanke (2012a) apresenta um estudo 
Quadro 1. Avanços recentes em Problemas de Roteirização e Estoques.

\begin{tabular}{|l|c|l|}
\hline \multicolumn{1}{|c|}{ Trabalho } & Política de Distribuição & \multicolumn{1}{c|}{ Principal Contribuição } \\
\hline Coelho e Laporte (2013) & $O U-M L-O T L$ & Nova Política de Distribuição \\
\hline Liu e Lee (2011) & $M L$ & Restrição de Janelas de Tempo \\
\hline Coelho, Cordeau e Laporte (2012b) & $O U-M L$ & Transbordo entre Clientes \\
\hline Coelho, Cordeau e Laporte (2012a) & $O U-M L$ & Consistência e Qualidade de Serviço \\
\hline Coelho, Laporte e Cordeau (2012) & $O U-M L$ & Transbordo com Demanda Estocástica \\
\hline Nishi e Izuno (2014) & $M L$ & Entregas Fracionadas \\
\hline
\end{tabular}

mais aprofundado. Contudo, a literatura ainda não apresenta um estudo comparativo de tais formulações com outras políticas de distribuição. No presente trabalho, o cálculo do lote econômico baseia-se em critérios dinâmicos, e segue o modelo básico de cálculo que considera o custo total do sistema de reposição (Cs), disponível em Schwarz (2008).

$$
C s_{i}=C_{C i} * D_{i}+C_{P i} *\left(\frac{D_{i}}{Q_{i}}\right)+C_{E_{i}} *\left(E_{m i}\right)
$$

Na equação (24), $C_{\mathrm{C}}$ é o custo de aquisição do produto, $D_{\mathrm{i}}$ é a demanda do período para o cliente $i, C_{\mathrm{Pi}}$ é o custo de processamento do pedido e $C_{\mathrm{Ei}}$ é o custo unitário de estocagem por período para o cliente $i$. A variável $E m_{\mathrm{i}}$ refere-se ao estoque médio do cliente $i$, enquanto $Q_{\mathrm{i}}$ define o lote econômico de entrega. Sem perda de generalidade, o custo de aquisição do produto $\left(C c_{\mathrm{i}}\right)$ pode ser desconsiderado, pois não impacta no custo do sistema de reposição.

\subsection{Política de Lote Econômico baseada nas Necessidades Líquidas (LE-NL)}

A demanda do cliente $i\left(D_{\mathrm{i}}\right)$ é calculada como uma demanda média por período, dada pelo conjunto de equações (25).

$$
D_{i}=\frac{1}{p} \sum_{t=1}^{p} d_{i}^{t}, \quad \forall i \in V^{\prime}
$$

$E m_{\mathrm{i}}$ é calculado como a média aritmética entre a quantidade recebida pelo cliente ao longo do horizonte de planejamento $p$ (assumindo que o cliente $i$ pode ser atendido em todos os períodos), e seu respectivo estoque médio por período (equação 26).

$$
E m_{i}=\frac{1}{2}\left(Q_{i} p+\frac{\sum_{t=1}^{p} I_{i}^{t}}{p}\right) \quad \forall i \in V^{\prime}
$$

O custo de processamento do pedido $\left(C p_{\mathrm{i}}\right)$ é obtido a partir da ponderação entre dois componentes: distância e quantidade transportada. Com relação à distância, o parâmetro ideal seria o comprimento do arco que conecta o cliente $i$ com o seu predecessor na rota. Como este predecessor não é conhecido a priori, considera-se como proxy o deslocamento médio entre todos os possíveis pontos de origem até o referido cliente $i$.

Nesta formulação, o conjunto de clientes potencialmente candidatos a serem pontos de origem para formar um arco com o cliente $i$ é delimitado pelo conjunto $S^{t}$. Um cliente $j \in S^{t}$ se a condição (27) for satisfeita.

$$
j \in S^{t} \mid d_{j}^{t}>\left(I_{j}^{t-1}-\min C_{j}\right), \forall j \neq i, \forall t \in T
$$

Assim, o custo de transporte para atender o cliente $i$ no período $t\left(\operatorname{dis}_{i}^{t}\right)$ é dado pelo deslocamento médio entre o cliente $i$ e todos os clientes que pertencem a $S^{t}$.

$$
\operatorname{dis}_{i}^{t}=\frac{1}{n_{t}^{\prime}} \sum_{j \in S} c_{i j}, \forall i \in V^{\prime}, \forall t \in T
$$

Em que $n_{t}{ }^{\prime}$ é a cardinalidade do conjunto $S^{t}$. Para um parâmetro independente do período, o componente da distância para o cliente $i\left(D i s_{i}\right)$ é definido pelo custo de transporte médio conforme (29).

$$
\operatorname{Dis}_{i}=\frac{1}{p} \sum_{t=1}^{T} \sum_{j \in S} t\left(\frac{c_{i j}}{n_{t}{ }^{\prime}}\right), \forall i \in V^{\prime}
$$

O segundo componente $C p_{\mathrm{i}}$ se refere à quantidade transportada. A partir de conceitos discutidos em Wanke (2012b), adota-se que, quanto maior for a fração de carga do cliente $i$ em relação à carga total transportada pela frota, maior é o custo de transporte associado. A fração de carga do cliente $i$ no período $t\left(r_{i}^{t}\right)$, é calculada pela razão entre a quantidade entregue ao cliente $i$ no período $t$ conforme política $M L\left[d_{i}^{t}-\left(I_{i}^{t-1}-\min C_{i}\right)\right]$ e a quantidade total entregue no período $\sum_{j \in S} d_{j}^{t}-\left(I_{j}^{t-1}-\min C_{j}\right)$,

$$
r_{i}^{t}=\frac{d_{i}^{t}-\left(I_{i}^{t-1}-\min C_{i}\right)}{\sum_{j \in S^{t}} d_{j}^{t}-\left(I_{j}^{t-1}-\min C_{j}\right)} \text {, tal que, } d_{i}^{t}>\left(I_{i}^{t-1}-\min C_{i}\right), \forall i \in V ; \forall t \in T
$$

É observado que o cliente $i$ terá sua fração de carga $r_{i}^{t}$ calculada no período $t$ se sua respectiva necessidade líquida for maior que zero. Expandindo $r_{i}^{t}$ para o horizonte de planejamento, calcula-se em (31) o componente de quantidade $\left(R_{\mathrm{i}}\right)$. 


$$
R_{i}=\frac{1}{p} \sum_{t=1}^{p}\left[\frac{d_{i}^{t}-\left(I_{i}^{t-1}-\min C_{i}\right)}{\sum_{j \in S} d_{j}^{t}-\left(I_{j}^{t-1}-\min C_{j}\right)}\right],
$$

tal que, $d_{i}^{t}>\left(I_{i}^{t-1}-\min C_{i}\right) \forall i \in V^{\prime}$

Finalmente, $C p_{\mathrm{i}}$ é calculado pelo produto de (29) e (31), ou seja, a ponderação entre o componente de distância $\left(D i s_{\mathrm{i}}\right)$ e o componente de quantidade $R_{\mathrm{i}}$ (equação 32).

$$
C_{P i}=\frac{1}{p^{2}} \sum_{t=1}^{p}\left[\sum_{j \in S} t\left(\frac{c_{i j}}{n_{t}{ }^{\prime}}\right)\right]\left[\frac{d_{i}^{t}-\left(I_{i}^{t-1}-\min C_{i}\right)}{\sum_{j \in S} d^{t}-\left(I_{j}^{t-1}-\min C_{j}\right)}\right] \text { (32) }
$$

tal que, $d_{i}^{t}>\left(I_{i}^{t-1}-\min C_{i}\right), \forall i \in V^{\prime}$

O custo do sistema de reposição $\left(C s_{\mathrm{i}}\right)$ no contexto do PRE é obtido pela substituição das equações (25), (26) e (32) na equação (25).

$$
C s_{i}=\frac{1}{p^{2}} \sum_{t=1}^{p}\left[\sum_{j \in S}\left(\frac{c_{i j}}{n_{t}}\right)\right]\left[\frac{d_{i}^{t}-\left(I_{i}^{t-1}-\min C_{i}\right)}{\sum_{j \in S} d_{j}^{t}-\left(I_{j}^{t-1}-\min C_{j}\right)}\right] *\left[\frac{1}{p_{t=1}^{p} d_{i}^{t}} Q_{i}\right]+h_{i} *\left[\frac{1}{2 p}\left(Q_{i} p+\sum_{t=1}^{p} I_{i}^{t}\right)\right](33)
$$

tal que, $d_{i}^{t}>\left(I_{i}^{t-1}-\min C_{i}\right), \forall i \in V^{\prime}$

Derivando (33) e resolvendo para $Q_{i}$, tem-se o lote econômico de entrega para o cliente $i$ conforme (34).

$$
Q_{i}=\sqrt{\frac{2\left[\frac{1}{p^{2}} \sum_{t=1}^{p} \sum_{j \in S^{t}}\left(\frac{c_{i j}}{n_{t}{ }^{\prime}}\right)\left(\frac{d_{i}^{t}-\left(I_{i}^{t-1}-\min C_{i}\right)}{\sum_{j \in S^{t}} d_{j}^{t}-\left(I_{j}^{t-1}-\min C_{j}\right)}\right]\left[\sum_{t=1}^{p} d_{i}^{t}\right]\right.}{h_{i}}}
$$

tal que, $d_{i}^{t}>\left(I_{i}^{t-1}-\min C_{i}\right), \forall i \in V^{\prime}$

A política implica que, se o cliente $i$ for visitado pelo veículo $k$ no período $t$, a quantidade recebida por este deve ser igual ou múltiplo do seu respectivo lote econômico $\left(Q_{\mathrm{i}}\right)$, limitado por sua capacidade de armazenagem $C_{i}$. A formulação para a política LE requer a adição da equação (35), modificando a equação (3) do modelo original, de forma que:

$$
q_{i k}^{t}=\min \left(\left(C_{i}-I_{i}^{t}\right) y_{i k}^{t} ; \theta_{i}^{t} * Q_{i}\right), \theta_{i}^{t}=\left\lceil\frac{d_{i}^{t}-\left(I_{i}^{t}-\min C_{i}\right)}{Q_{i}}\right\rceil(35)
$$

Em que $\theta_{i}^{t}$ é o maior valor inteiro ("teto") da divisão entre a quantidade necessária a ser entregue ao cliente $i$ (necessidade líquida) e o próprio lote econômico $Q_{\mathrm{i}}$.

\subsection{Política Lote Econômico baseada nas Necessidades Brutas (LE-NB)}

A formulação LE-NB desconsidera/considera o estoque do cliente para o cômputo do $C p_{i}$. Dessa maneira, $S^{t}$ é dado pelo próprio conjunto $V$. O custo de transporte para atender o cliente $i$ independente do período $\left(D i s_{\mathrm{i}}\right)$ fica sendo:

$$
D i s_{i}=\frac{1}{n}\left(\sum_{j=0, j \neq i}^{n} c_{i j}\right), \forall i \in V^{\prime}
$$

A fração de carga para o cliente $i$ também independe do período e é determinada pela parcela da demandada pelo cliente ao longo do horizonte $p$ em relação à demanda total de $V^{\prime}$.

$$
R_{i}=\frac{1}{\sum_{i=1}^{n} \sum_{t=1}^{p} d_{i}^{t}}\left(\sum_{t=1}^{p} d_{i}^{t}\right), \forall i \in V^{\prime}
$$

O custo de processamento do pedido do cliente $i$ permanece sendo o produto entre $R_{\mathrm{i}}$ e $D i s_{\mathrm{i}}$. Tanto a demanda, estoque médio e custo de estocagem são idênticos à formulação LE-NL. O custo do sistema de reposição é dado pela equação (38).

$$
C s_{i}=\frac{1}{n p Q_{i} \sum_{i=1}^{n} \sum_{t=1}^{p} d_{i}^{t}}\left[\sum_{j=0, j \neq i}^{n} c_{i j}\left(\sum_{t=1}^{p} d_{i}^{t}\right)^{2}\right]+\frac{h_{i}}{2 p}\left[Q_{i} p+\sum_{t=1}^{p} I_{i}^{t}\right], \forall i \in V^{\prime}
$$

Derivando (38) e resolvendo para $Q_{\mathrm{i}}$, tem-se o lote econômico conforme equação (39). A formulação para o modelo da seção 2 segue a equação (35).

$$
Q_{i}=\sqrt{\frac{2\left[\sum_{j=0, j \neq i}^{n} c_{i j}\left(\sum_{t=1}^{p} d_{i}^{t}\right)^{2}\right]}{h_{i}(n p)^{2} \sum_{i=1}^{n} \sum_{t=1}^{p} d_{i}^{t}}}, \forall i \in V^{\prime}
$$

\subsection{Formulação da política $E O Q$ baseada}

\section{em Distâncias (LE-D)}

Esta terceira proposta atende aos mesmos parâmetros da LE-NB, exceto por desconsiderar a fração de carga, fazendo com que o custo de processamento do pedido seja idêntico a (36). A equação que determina o custo do sistema de reposição do cliente $i$ é apresentado em (40).

$$
C s_{i}=\frac{1}{n p Q_{i}}\left[\sum_{j=0, j \neq i}^{n} c_{i j} \sum_{t=1}^{p} d_{i}^{t}\right]+\frac{h_{i}}{2 p}\left[Q_{i} p+\sum_{t=1}^{p} I_{i}^{t}\right], \forall i \in V^{\prime}
$$

O lote econômico de entrega para LE-D descrito em (41) é obtido pela derivada de (40) em relação a $Q_{\mathrm{i}}$ e resolvido para este mesmo termo. A modelagem também segue (36).

$$
Q_{i}=\sqrt{\frac{2 \sum_{j=0, j \neq i}^{n} c_{i j} \sum_{t=1}^{p} d_{i}^{t}}{h_{i} n p}}, \forall i \in V^{\prime}
$$

\section{Estratégia de resolução}

A solução do PRE é realizada em dois estágios. Primeiramente efetua-se programação das entregas 
para o conjunto de clientes ao longo de $p$, conforme a política de distribuição ( $M L, O U$ ou LE). Dessa maneira, a cada período $t$, um conjunto de clientes que serão atendidos $\left(E^{t}\right)$ é construído. O cliente $i$ irá pertencer a $E^{t}$ caso não possua estoque disponível para atender a sua demanda no período $t$, isto é, $d_{i}^{t}>\left(I_{i}^{t-1}-\min C_{i}\right)$. A quantidade a ser entregue a este cliente $\left(q_{i}^{t}\right)$ é dada conforme os critérios de cada política, sendo:

\section{Maximum Level}

$$
q_{i}^{t}=d_{i}^{t}-\left(I_{i}^{t-1}-\min C_{i}\right), \forall i \in V^{\prime}, \forall t \in T
$$

\section{Order-up-to-level}

$$
q_{i}^{t}=C_{i}-\left(I_{i}^{t-1}-\min C_{i}\right), \forall i \in V^{\prime}, \forall t \in T
$$

\section{Lote Econômico}

$$
q_{i}^{t}=\min \left(\left(C_{i}-I_{i}^{t}\right) y_{i k}^{t} ; \theta_{i}^{t} * Q_{i}\right), \theta_{i}^{t}=\left[\frac{d_{i}^{t}-\left(I_{i}^{t}-\min C_{i}\right)}{Q_{i}}\right], \forall i \in V^{\prime}, \forall t \in T(44)
$$

Definidos $E^{t}$ e $q_{i}^{t}$, PRE é decomposto em $p$ problema de roteirização de veículos (PRVC), um para cada instante $t(t=1, \ldots, p)$.

Dentre as diversas possibilidades de resolução, o PRVC pode ser abordado também em dois estágios (POP; KARA; MARC, 2012; NANANUKUL, 2013). Neste trabalho, os clientes são agrupados em até $K$ grupos, de acordo com sua demanda e posição geográfica, sendo um grupo para cada veículo disponível na frota $(k=1, \ldots, K)$. Garante-se que a quantidade a ser entrega a cada grupo $k$ não excede a capacidade de carregamento do veículo $(Q)$. Dessa maneira, o PRVC recai em até $K$ Problemas de Caixeiro Viajante simples (PCVs).

\subsection{Problema de agrupamento dos pontos de demanda no contexto do PRE}

O agrupamento dos pontos de demanda pode ser formulado como uma variante do Problema das $p$-Medianas Capacitado (PPMC) (DÍAZ; FERNÁNDEZ, 2006), demandando a localização de $p$-facilidades dentre um grupo de pontos candidatos. Cada $p$-facilidade locada possui uma capacidade de forma a atender à demanda de um conjunto de clientes designados a ela. O problema é modelado como segue:

Seja $E^{t}=\left\{1, \ldots, n^{t}\right\}$ o conjunto formado pelos clientes com entregas programadas no período $t \mathrm{e}$ $K$, com $k=\{1, \ldots, K\}$, o conjunto de candidatos para as facilidades (frota de veículos). Cada facilidade possui a mesma capacidade de carregamento $Q$ de um veículo da frota $\mathrm{O}$ cliente $i \in E^{t}$ possui uma coordenada cartesiana determinada por $\left(x_{i}, y_{i}\right)$ e uma quantidade a ser entregue no período $t$ dada por $q_{i}^{t}$ calculada a priori pela política de distribuição. O objetivo do problema é determinar, para cada $k$ facilidade, uma localização $\left(x_{k}, y_{k}\right)$, de forma que a soma das distâncias euclidianas $d_{i k}=\sqrt{\left(x_{i}-x_{k}\right)^{2}+\left(y_{i}-y_{k}\right)^{2}}$ entre todos os pontos de $E^{t}$ às $k$ facilidades $(k \in K)$ seja mínimo. As variáveis de decisão do problema são definidas por $w_{i k}$, que assume o valor 1 se o cliente $i$ é designado para a facilidade $k$ e zero caso contrário; e $g_{k}$, que assume o valor 1 se a facilidade $k$ for instalada e zero caso contrário. O problema é modelado como segue:

Minimizar:

$$
Z=\sum_{i=1}^{n} \sum_{k=1}^{K}\left(\sqrt{\left(x_{i}-x_{k}\right)^{2}+\left(y_{i}-y_{k}\right)^{2}}\right) * w_{i k}
$$

Sujeito a:

$$
\begin{aligned}
& \sum_{k=1}^{K} w_{i k}=1 \quad \forall i \in V^{t} \\
& \sum_{i=1}^{n^{t}} g_{k} \leq K \quad \forall k \in K \\
& \sum_{i=1}^{n^{t}} w_{i k} q_{i}^{t} \leq Q_{k} \quad \forall k \in K \\
& \sum_{i=1}^{n^{t}} w_{i k} \leq N \quad \forall k \in K \\
& w_{i k}, g_{k} \in\{0,1\} \quad \forall k \in K \quad \forall i \in E^{t} \\
& x_{k} \geq 0 \quad \forall k \in K \\
& y_{k} \geq 0 \quad \forall k \in K
\end{aligned}
$$

Em (46) a função objetivo é minimizada, dada pelo somatório das distâncias entre os clientes e as $k$ facilidades. Já o conjunto de restrições (47) garante que um cliente seja designado para apenas uma facilidade. As restrições em (48) impedem que o número de facilidades extrapole a disponibilidade da frota. Em (49) são garantidos que as facilidades não tenham sua capacidade violada. $O$ conjunto de restrições (50) limita o número de clientes designado por facilidade de acordo com o limitante máximo introduzido na equação (9). O conjunto de restrições de (51) até (53) delimita o domínio das variáveis de decisão.

\subsubsection{Algoritmo heurístico para agrupamento dos pontos de demanda}

A definição dos grupos de atendimento é realizada/ obtida por meio da técnica estatística k-means, em que o número de grupos é igual ao número de veículos disponíveis. Reportam-se na literatura alguns estudos que aplicam a técnica $k$-means em problemas de agrupamento e roteirização (KAVEH; ZADEH; SAHRAEIAN, 2010; GEETHA; POONTHALIR; VANATHI, 2009; LIAO; GUO, 
2008) com resultados bastante satisfatórios, justificando o uso desse algoritmo.

Neste estudo, são geradas inicialmente as coordenadas aleatórias para $K$ sementes e os clientes de $E^{\mathrm{t}}$ são então designados de acordo com a proximidade e com a quantidade programada de entrega, respeitando a limitação de carregamento do veículo e o número máximo $N$ de clientes por grupo. Definido um arranjo inicial, novas coordenadas são calculadas para os $K$-centroides pela razão entre a quantidade total alocada ao grupo e a soma das distâncias entre as coordenadas de todos os clientes e a coordenada do centroide do grupo. A partir de então os clientes são designados novamente. O procedimento é repetido até que as novas coordenadas dos centroides convirjam para um limite previamente estabelecido. O Quadro 2 apresenta o algoritmo k-means adaptado para este trabalho.

A modificação no algoritmo k-means clássico reside na limitação do número de pontos por cluster, respeitando a restrição operacional proposta na equação (8). Cabe destacar que o processo é realizado a cada período de planejamento $t$, dinamizando a estratégia de partição de conjunto fixo (ANILY; BRAMEL, 2004; LI; CHEN; CHU, 2010; ZHAO; CHEN; ZANG, 2008). Depois da definição dos grupos de atendimento, a etapa seguinte constrói os roteiros para os grupos formados.

\subsection{Roteirização dos grupos formados}

O processo de roteirização consiste na resolução de $k$-Problemas do Caixeiro Viajante (PCV), uma vez que os grupos de atendimento já foram definidos na etapa anterior. Para cada um dos PCVs, a técnica de resolução se desenvolve em duas etapas. Inicialmente são utilizadas heurísticas construtivas para a geração das rotas iniciais. Na segunda etapa, o roteiro inicial é refinado.

Neste trabalho, optou-se pelo algoritmo da inserção mais econômica, proposto incialmente Rosenkrantz, Stearns e Lewis (1977). Esta heurística atende a um conjunto de critérios que garante certa eficiência para a etapa de refinamento posterior. $\mathrm{O}$ algoritmo consiste em, a partir de um arco $(i, j)$, encontrar um nó $k \in V$ que ainda não esteja na rota de maneira que $c_{\mathrm{ik}}+c_{\mathrm{kj}}-c_{\mathrm{ij}}$ seja mínimo e formar a rota $(i, k, j)$ até se ter um circuito Hamiltoniano.

No caso dos algoritmos de melhorias em uma rota, o refinamento da solução incumbente busca reduzir a distância total percorrida advinda de uma solução inicial obtida a priori. Uma das estratégias mais comuns são os algoritmos de trocas de arcos $k$-opt, devido à Lin e Kernighan (1973). Dentre as técnicas mais utilizadas, destacam-se as trocas 2-opt.

A aplicação das trocas 2-opt no contexto do PRE pode ser encontrada nos trabalhos de Popović, Vidović e Radivojević (2012), Boudia e Prins (2009), Liu e Chen (2012) e, mais recentemente, em Shukla et al. (2013). Um pseudocódigo é apresentado em Chaves (2009).

\subsection{Melhoria entre rotas por técnicas de Busca em Vizinhança Ampla (BVA)}

Heurísticas baseadas em Busca em Vizinhança Ampla (BVA) vêm demonstrando bons resultados em vários problemas de otimização em transporte

Quadro 2. Algoritmo k-means.

\begin{tabular}{|c|l|}
\hline \multicolumn{2}{|c|}{ Algoritmo $\boldsymbol{k}$-means (Programação das Entregas) } \\
\hline $\mathbf{1}$ & Ler Dados \\
\hline $\mathbf{2}$ & Melhor Solução $\leftarrow 1000^{\wedge} 1000$ \\
\hline $\mathbf{3}$ & Solução Atual $\leftarrow 0$ \\
\hline $\mathbf{4}$ & Gerar k Centroides $\left(x_{k}, y_{k}\right)$ \\
\hline $\mathbf{5}$ & Enquanto (Melhor Solução - Solução Atual) > Tolerância \\
\hline $\mathbf{6}$ & Para i=1 até $\boldsymbol{n}^{t}$ \\
\hline $\mathbf{7}$ & Alocar $i$ no centroide $\mathbf{k}$, tal que $\sqrt{\left(x_{i}-x_{k}\right)^{2}+\left(y_{i}-y_{k}\right)^{2}}$ seja mínimo e $\sum_{i=1}^{n^{t}} w_{i k} \leq N, \sum_{i=1}^{n^{t}} w_{i k} q_{i}^{t} \leq Q_{k}$ \\
\hline $\mathbf{8}$ & Fim Para \\
\hline $\mathbf{9}$ & Para $\mathbf{k}=\mathbf{1}$ até K \\
\hline $\mathbf{1 0}$ & Recalcular $\left(x_{k}, y_{k}\right)$ \\
\hline $\mathbf{1 0}$ & Fim Para \\
\hline $\mathbf{1 1}$ & Solução Atual $\leftarrow \sum_{i=1}^{n^{t}} \sum_{k=1}^{K}\left(\sqrt{\left(x_{i}-x_{k}\right)^{2}+\left(y_{i}-y_{k}\right)^{2}}\right) w_{i k}$ \\
\hline $\mathbf{1 2}$ & Fim Equanto \\
\hline $\mathbf{1 3}$ & Return $($ Grupos de Atendimento) \\
\hline
\end{tabular}


e logística, com destaque em Goel et al. (2012), Adulyasak, Cordeau e Jans (2012), Schmid, Doerner e Laporte (2013), Coelho (2012) e Coelho et al. (2012c). A técnica foi proposta por Shaw (1998), e o processo de melhoria a partir de uma solução inicial se desenvolve pela alternância entre destruição e reparação desta solução. Em Pisinger e Ropke (2010), é apresentado um estudo detalhado sobre algoritmos do tipo BVA, com o devido rigor matemático. Conforme os autores, um dos pontos-chave é a estratégia utilizada para definir a dimensão do espaço de busca na vizinhança de uma solução incumbente. Particularmente no caso da BVA, a dimensão de busca é delimitada pelos operadores de destruição e reparação da solução inicial. Em problemas de roteirização, um operador de destruição consiste na eliminação de certa quantidade de vértices ou arcos de uma solução incumbente, conforme taxa (percentual em relação aos pontos roteirizados) estabelecida a priori. Já o operador de reparação atua na reinserção desses vértices ou arcos no roteiro destruído, a fim de se encontrar uma solução de menor custo.

\subsubsection{Operador de Destruição}

A partir de uma solução inicial para o PRE em um dado período $t$, o operador de destruição seleciona, respeitando a taxa de destruição de pontos previamente estabelecida, os clientes que possuem as maiores diferenças entre a distância ao centroide de sua respectiva rota e ao cliente mais próximo que pertença à outra rota. Dessa forma, cria-se um conjunto de pontos que serão destruídos, ordenados de forma decrescente por essa diferença.

Em geral, os cruzamentos entre rotas são intensificados por clientes que estão relativamente distantes do centro da rota. Ao mesmo tempo, tais clientes distantes possuem maior proximidade com clientes de outras rotas. É o caso do cliente 3 (em destaque) que pertence à rota 1 (Figura 2). Nota-se que ele se encontra mais próximo $\left(\right.$ Dist $_{2}<$ Dist $\left._{1}\right)$ do cliente 8 (em destaque) que pertence à rota 2 que $o$ centroide da rota 1 .

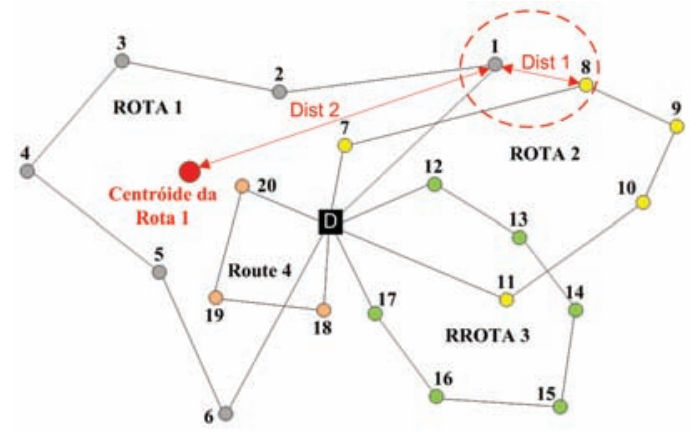

Figura 2. Ilustração de "Ponto destruído". Fonte: Adaptado de Pisinger e Ropke (2010).
Conforme o exemplo, clientes que possuem as maiores diferenças ( Dist $_{2}-$ Dist $_{1}$ ), respeitando o limite do número de pontos destruídos serão removidos da solução incumbente para, posteriormente, serem realocados em outras rotas conforme definido pelo operador de reparação.

\subsubsection{Operador de reparação}

Sequencialmente à destruição dos pontos, o operador de reparação insere o conjunto de pontos removidos na rota em que este possui a maior proximidade com outro cliente. Atenta-se que a inserção só ocorrerá se o veículo designado para a rota que receberá o ponto dispuser de capacidade para atender à quantidade que deve ser entregue a este cliente e, ainda, se o número máximo de pontos por rota não for violado. O ponto é inserido no final do roteiro e, para otimizar a nova rota formada, o procedimento de melhoria 2-opt é novamente operado sobre toda rota que for alterada, seja com a remoção de clientes ou com a inserção deles. O Quadro 3 apresenta o procedimento de BVA desenvolvido neste trabalho.

\subsection{Heurística proposta}

A heurística proposta envolve a junção dos algoritmos discutidos nas seções anteriores. Como as demandas são conhecidas de antemão, o primeiro passo da heurística consiste em programar as entregas conforme as três políticas de distribuição consideradas - Maximum Level, Order-up-to-level e Lote Econômico - para todo o horizonte de planejamento. Sequencialmente, para cada período $t \in T$, os grupos de atendimento são gerados para atender ao conjunto de clientes programados para $o$ período. Emprega-se para tal o algoritmo k-means modificado, atentando para o número máximo de clientes por rota, formando até $K$ grupos, sendo um para cada veículo $k$ disponível.

O terceiro passo constrói as rotas para os grupos formados, sendo esta etapa procedida pela aplicação das trocas 2-opt em todas as rotas geradas. Por fim, aplica-se a melhoria BVA entre todos os roteiros existentes. O processo é retomado a cada novo período de planejamento. O Quadro 4 apresenta a estrutura completa.

\section{Cenários gerados e resultados obtidos}

Os cenários gerados seguem os padrões adotados em Coelho, Laporte e Cordeau (2012) e Archetti et al. (2007). As variáveis consideram a demanda dos clientes, níveis iniciais de estoque e localização geográfica dos vértices (clientes e depósito). O 
Quadro 3. Busca em Vizinhança Ampla Proposta.

\begin{tabular}{|c|l|}
\hline \multicolumn{2}{|c|}{ Algoritmo BVA (Solução Incumbente) } \\
\hline $\mathbf{1}$ & Ler Dados \\
\hline $\mathbf{2}$ & MaxP $\leftarrow$ Taxa de Destruição $* V$ \\
\hline $\mathbf{3}$ & Solução Analisada $\leftarrow$ Solução Incumbente \\
\hline \multirow{2}{*}{$\mathbf{3}$} & Para $i=1$ até MaxP Faça \\
\cline { 2 - 3 } & Para $k=1$ até $K$ Faça \\
\hline $\mathbf{4}$ & Diferença $i=$ Distância Centro Rota $k-$ Distância Cliente mais Próximo $\notin k$ \\
\hline $\mathbf{5}$ & Vetor Destruído $(i)$ \\
\hline $\mathbf{6}$ & Ordenar Vetor Destruído $(i)$ \\
\hline $\mathbf{7}$ & Fim Para \\
\hline $\mathbf{8}$ & Fim Para \\
\hline $\mathbf{9}$ & Para $i=1$ até MaxP Faça \\
\hline $\mathbf{1 0}$ & Inserir Cliente $i$ na rota do Cliente mais Próximo $\notin k$ \\
\hline $\mathbf{1 1}$ & Aplicar Melhoria 2 -opt \\
\hline $\mathbf{1 2}$ & Fim Para \\
\hline $\mathbf{1 3}$ & Se Custo (Solução Analisada) $<$ Custo (Solução Incumbente) então \\
\hline $\mathbf{1 4}$ & Solução Incumbente $\leftarrow$ Solução Analisada \\
\hline $\mathbf{2 0}$ & Fim Se \\
\hline $\mathbf{2 2}$ & Return (Solução Incumbente) \\
\hline
\end{tabular}

conjunto de instâncias é definido pelos parâmetros a seguir:

- Número de clientes $(n): 5,10,15,25,50,75$ e 100

- Número de veículos $(K): 2,3$ e 4 veículos

- Horizonte de Planejamento (p): 5, 10 e 20 períodos

- Demanda $\left(d_{\mathrm{i}}\right)$ : variável uniforme contínua entre [10;100]

- Taxa de recarga do depósito (r): variável uniforme contínua entre [100n,140n] em que $n$ é o número de clientes

- Taxa de destruição de pontos em uma rota (para a BVA): 0,4

- Distância do CD a BF: $\xi=1$

- Capacidade de Estocagem $\left(C_{\mathrm{i}}\right)$ :variável uniforme delimitada por $\left[2,6 \mu_{\mathrm{i}} ; 5,2 \mu_{\mathrm{i}}\right]$ em que:

$\mu_{i}=\frac{1}{p} \sum_{t=1}^{p} d_{i}^{t}, t \in T, i \in V$

- Percentual Mínimo de Estocagem $\left(\min C_{\mathrm{i}}\right): \alpha C_{\mathrm{i}}$, em que $\alpha$ é uma variável uniforme contínua entre $[0,1 ; 0,2]$

- Estoque Inicial no Depósito

$\left(I_{0}^{0}\right): \sum_{t=1}^{p} C_{i}$

- Estoque Inicial nos Clientes

$\left(I_{i}^{0}\right): C_{i}-\left(\frac{1}{p} \sum_{t=1}^{p} d_{i}^{t}\right)$
- Custo de estocagem do fornecedor $\left(h_{0}\right): 0,02$

- Custo de estocagem do cliente $\left(h_{\mathrm{i}}\right)$ :

Baixo Custo de Estocagem: Variável uniforme contínua entre $[0,02 ; 0,05]$

Alto Custo de Estocagem: Variável uniforme contínua entre $[0,2 ; 0,5]$

- Número Máximo de Clientes por Rota $N$ : parte inteira de $n / K$

- Capacidade do Veículo $(Q)$ :

$\left(\frac{5}{4}\right) *\left(\frac{1}{K} \sum_{i=1}^{n} C_{i}\right)$

- Distância entre os $\operatorname{arcos}(i, j) \quad\left(c_{\mathrm{ij}}\right)$ : $\sqrt{\left(X_{i}-X_{j}\right)^{2}+\left(Y_{i}-Y_{j}\right)^{2}}$, em que:

Baixo Custo de Transporte: $\left(X_{\mathrm{i}}, Y_{\mathrm{i}}\right)$ são variáveis uniformes entre [0;100].

Médio Custo de Transporte: $\left(X_{\mathrm{i}}, Y_{\mathrm{i}}\right)$ são variáveis uniformes entre [0;500].

Alto Custo de Transporte: $\left(X_{\mathrm{i}}, Y_{\mathrm{i}}\right)$ são variáveis uniformes entre [0;1000].

Foram consideradas 6 configurações de custos, sendo: $L t L i$ - Baixo Custo de Transporte e Baixo Custo de Estoque; $\mathrm{LtHi}$ - Baixo Custo de Transporte e Alto Custo de Estoque; $M t L i$ - Médio Custo de Transporte e Baixo Custo de Estoque; $\mathrm{MtHi}$ - Médio Custo de Transporte e Alto Custo de Estoque; $\mathrm{HtLi}$ - Alto Custo de Transporte e Baixo Custo de Estoque; e $\mathrm{HtHi}$ - Alto Custo de Transporte e Alto Custo de Estoque.

Para cada uma das configurações de custo, foram geradas 7 quantidades diferentes de clientes, com 
Quadro 4. Heurística proposta para o PRE.

\begin{tabular}{|l|l|}
\hline \multicolumn{2}{|c|}{ Heurística para o MIRP (Dados) } \\
\hline $\mathbf{1}$ & Ler Dados \\
\hline $\mathbf{3}$ & Para $t=l$ até $T$ Faça \\
\hline $\mathbf{4}$ & Programar Entregas (ML - OU - LE) \\
\hline $\mathbf{5}$ & Fim Para \\
\hline $\mathbf{6}$ & Para $t=l$ até $T$ Faça \\
\hline 7 & Para $k=l$ até $K$ Faça \\
\hline $\mathbf{8}$ & Agrupar Clientes (Algoritmo K-means) \\
\hline $\mathbf{9}$ & $\begin{array}{l}\text { Construir Roteiro Inicial (Inserção Mais } \\
\text { Econômica) }\end{array}$ \\
\hline $\mathbf{1 0}$ & Aplicar melhoria 2-opt \\
\hline $\mathbf{1 1}$ & Fim Para \\
\hline $\mathbf{1 2}$ & Aplicar melhoria BVA \\
\hline $\mathbf{1 3}$ & Fim Para \\
\hline $\mathbf{1 4}$ & Return (Solução) \\
\hline
\end{tabular}

3 diferentes quantidades de veículos e 3 quantidades diferentes de períodos, totalizando $(7 * 3 * 3)=63$ instâncias. Levando em conta as 6 combinações de custos, o número de cenários gerados foi de 378 .

Os experimentos computacionais foram realizados em um processador Intel Core i $3^{\mathrm{TM}}$ de 32 bits, CPU de $3.10 \mathrm{GHz}$ com 2GB de memória, sistema operacional Windows 7 Home, Service Pack 1. A heurística proposta foi implementada em linguagem Visual Basic.NET. Foram utilizados dois critérios de parada: cada teste computacional foi limitado em 100 iterações para instâncias com até 50 clientes e 10 iterações para instâncias com mais de 50 clientes, não podendo ultrapassar 60 minutos por teste. Cada iteração é configurada por uma nova geração aleatória de sementes durante o processo de agrupamento dos pontos de demanda.

\subsection{Resultados obtidos}

A Tabela 1 apresenta a primeira síntese dos resultados obtidos. Por razões de espaço, são apresentados os valores médios para cada uma das 6 configurações de custos consideradas. Ressalta-se que para cada uma delas, foram processadas 63 instâncias com variações quanto ao número de clientes, períodos e veículos conforme informado na seção 5. Da esquerda para a direita, tem-se nas duas primeiras colunas as configurações de custos. Sequencialmente é apresentada a melhor solução média conhecida acompanhada do custo médio de transporte (CTr), custo médio de estocagem no fornecedor (CEstF), do custo médio de estocagem nos clientes (CEstC), do custo médio total (CT) e do tempo médio de processamento em segundos ( $\mathrm{T}(\mathrm{s})$ ).

Em negrito, estão destacados os menores valores médios observados para as diferentes combinações de custos analisadas. Para o custo médio de transporte, é possível observar o predomínio da política $O U$ sobre as configurações $L t-L i, L t-H i$, $\mathrm{Mt}-\mathrm{Hi}$ e $\mathrm{Hi}$-Hi. A política LE-D apresenta um custo médio de transporte menor para as configurações $M t-L i, H i-L i$. Como esperado, a política $M L$ detém os menores custos médios de estocagem no cliente, pois está endereçada à minimização do estoque, enquanto a política $O U$, por transportar maiores quantidades, acaba consumindo proporções maiores do volume reabastecido no fornecedor a partir da $\mathrm{BF}$ (parâmetro $r$ ), minimizando o custo de estocagem no fornecedor. Com relação ao custo médio total (CT), a política LE-D possui desempenho médio superior para as configurações $M t-L i$ e $H i-L i$, devido, em grande parte, ao reduzido custo de transporte que acabou produzindo nestas configurações de custos. A política ML destacou-se na configuração de custos mais favorável a ela $(\mathrm{Li}-\mathrm{Hi})$, dado que a proximidade dos clientes (reduzido custo de transporte - $\mathrm{Li}$ ) associada ao elevado custo de estocagem $(H i)$ favorece visitas mais frequentes em detrimento ao estoque do produto no cliente. Para as demais configurações, prevalece o predomínio da política $O U$. Evidencia-se que as formulações de lote econômico por necessidades líquidas (LE - NL) e necessidades brutas (LE - NB) não apresentam domínio sobre as demais políticas. O desempenho médio superior da política $O U$, entretanto, não é verificado na análise global para os 378 cenários. Destaca-se que o menor desempenho médio global foi obtido pela política LE-D, com 34421 diante de 34978 da política $O U$. A Figura 3 ilustra a discussão graficamente. Com relação ao tempo de processamento médio, as cinco políticas de distribuição analisadas demandam valores próximos a 100 segundos para a resolução de um cenário. Fica demonstrado, portanto, que a estratégia heurística é bastante competitiva neste quesito.

A verificação dos resultados pelos valores médios é mais elucidada quando se compara o número de vitórias (menor custo total) por cenário. A Tabela 2 apresenta esses valores.

Embora a política OU tenha produzido o menor resultado médio para a configuração Lt-Li, a Tabela 2 aponta que a política LE-D produziu um custo total menor para 32 dos 63 cenários. Outrossim, ressalta-se o desempenho da política ML para a configuração Lt-Hi, que apresentou desempenho superior para 52 cenários. A Tabela 2 também possibilita concluir que as formulações LE-NL e LE-NB foram vitoriosas em 21 e 10 cenários na configuração Ht-Li. Indica-se em negrito a política de distribuição para cada uma das seis configurações de custos. A formulação LE-D produziu melhores resultados para aproximadamente $40 \%$ dos cenários testados, vencendo em 148 deles. Salienta-se que o número de vitórias por configuração pode 
Tabela 1. Resumo dos Resultados.

\begin{tabular}{|c|c|c|c|c|c|c|c|c|c|c|c|c|}
\hline \multirow{2}{*}{ C.Trp. } & \multirow{2}{*}{ C. Est. } & \multirow{2}{*}{$\begin{array}{c}\text { Média } \\
\text { BKS }\end{array}$} & \multicolumn{5}{|c|}{ Maximum Level } & \multicolumn{5}{|c|}{ Order-up-to-level } \\
\hline & & & $\mathrm{CTr}$ & CEstF & CEstC & CT & $\mathbf{T}(\mathrm{s})$ & $\mathrm{CTr}$ & CEstF & CEstC & CT & $\mathbf{T}(\mathbf{s})$ \\
\hline $\mathrm{Lt}$ & $\mathrm{Li}$ & 7047 & 7249 & 717 & 451 & 8417 & 82 & 5249 & 650 & 1230 & 7128 & 104 \\
\hline $\mathrm{Lt}$ & $\mathrm{Hi}$ & 12543 & 7300 & 696 & 4610 & 12606 & 103 & 5246 & 626 & 12526 & 18400 & 124 \\
\hline Mt & $\mathrm{Li}$ & 27161 & 35946 & 719 & 460 & 37125 & 104 & 25551 & 637 & 1251 & 27445 & 96 \\
\hline Mt & $\mathrm{Hi}$ & 37403 & 35724 & 695 & 4542 & 40961 & 114 & 26053 & 629 & 12404 & 39124 & 128 \\
\hline $\mathrm{Hi}$ & $\mathrm{Li}$ & 53068 & 71988 & 720 & 454 & 73163 & 100 & 52307 & 644 & 1234 & 54205 & 152 \\
\hline $\mathrm{Hi}$ & $\mathrm{Hi}$ & 62859 & 71055 & 701 & 4563 & 76318 & 127 & 50306 & 635 & 12453 & 63569 & 107 \\
\hline Média & Global & 33347 & 38210 & 708 & 2513 & 41432 & 105 & 27452 & 636.9 & 6850 & 34978 & 119 \\
\hline \multirow{2}{*}{ C.Trp. } & \multirow{2}{*}{ C. Est. } & \multirow{2}{*}{$\begin{array}{c}\text { Média } \\
\text { BKS }\end{array}$} & \multicolumn{5}{|c|}{ Lote Econômico - NL } & \multicolumn{5}{|c|}{ Lote Econômico - NB } \\
\hline & & & $\mathrm{CTr}$ & CEstF & CEstC & CT & $\mathbf{T}(\mathbf{s})$ & CTr & CEstF & CEstC & CT & $\mathbf{T}(\mathbf{s})$ \\
\hline $\mathrm{Lt}$ & $\mathrm{Li}$ & 7047 & 6178 & 676 & 764 & 7619 & 144 & 7050 & 699 & 566 & 8315 & 132 \\
\hline $\mathrm{Lt}$ & $\mathrm{Hi}$ & 12543 & 7091 & 662 & 5763 & 13519 & 122 & 7289 & 682 & 5064 & 13037 & 103 \\
\hline Mt & $\mathrm{Li}$ & 27161 & 26834 & 660 & 1015 & 28515 & 145 & 32025 & 688 & 692 & 33412 & 91 \\
\hline Mt & $\mathrm{Hi}$ & 37403 & 32159 & 664 & 6882 & 39705 & 124 & 35151 & 677 & 5410 & 41239 & 131 \\
\hline $\mathrm{Hi}$ & $\mathrm{Li}$ & 53068 & 52282 & 671 & 1105 & 54080 & 137 & 61432 & 675 & 770 & 62899 & 124 \\
\hline $\mathrm{Hi}$ & $\mathrm{Hi}$ & 62859 & 60099 & 644 & 7719 & 68636 & 101 & 69073 & 664 & 5724 & 75635 & 117 \\
\hline Média & Global & 33347 & 30774 & 662.7 & 3875 & 35346 & 129 & 35337 & 680.8 & 3038 & 39089 & 116 \\
\hline \multirow{2}{*}{ C.Trp. } & \multirow{2}{*}{ C. Est. } & Média & \multicolumn{5}{|c|}{ Lote Econômico - SD } & & & & & \\
\hline & & BKS & CTr & CEstF & CEstC & CT & $\mathbf{T}(\mathrm{s})$ & & & & & \\
\hline $\mathrm{Lt}$ & $\mathrm{Li}$ & 7047 & 5464 & 661 & 1093 & 7218 & 121 & & & & & \\
\hline $\mathrm{Lt}$ & $\mathrm{Hi}$ & 12543 & 6760 & 646 & 7207 & 14617 & 120 & & & & & \\
\hline Mt & $\mathrm{Li}$ & 27161 & 25466 & 650 & 1197 & 27319 & 99 & & & & & \\
\hline $\mathrm{Mt}$ & $\mathrm{Hi}$ & 37403 & 28926 & 633 & 9779 & 39338 & 117 & & & & & \\
\hline $\mathrm{Hi}$ & $\mathrm{Li}$ & 53068 & 51649 & 665 & 1181 & 53516 & 130 & & & & & \\
\hline $\mathrm{Hi}$ & Hi & 62859 & 52683 & 647 & 11014 & 64518 & 126 & & & & & \\
\hline Média & Global & 33347 & 28491 & 650.2 & 5245 & 34421 & 119 & & & & & \\
\hline
\end{tabular}

Tabela 2. Número de Vitórias.

\begin{tabular}{|c|c|c|c|c|c|c|}
\hline C.Trp. & C.Est. & ML & $\mathrm{OU}$ & LE-NL & LE-NB & LE-D \\
\hline $\mathrm{Lt}$ & $\mathrm{Li}$ & 0 & 28 & 5 & 1 & 32 \\
\hline $\mathrm{Lt}$ & $\mathrm{Hi}$ & 52 & 6 & 3 & 0 & 2 \\
\hline Mt & $\mathrm{Li}$ & 0 & 19 & 16 & 2 & 35 \\
\hline Mt & $\mathrm{Hi}$ & 15 & 25 & 7 & 0 & 16 \\
\hline $\mathrm{Ht}$ & $\mathrm{Li}$ & 0 & 11 & 21 & 10 & 36 \\
\hline $\mathrm{Ht}$ & $\mathrm{Hi}$ & 2 & 28 & 6 & 0 & 27 \\
\hline \multicolumn{2}{|c|}{ TOTAL } & 69 & 117 & 58 & 13 & 148 \\
\hline
\end{tabular}

Tabela 3. Desvios em relação à menor média conhecida.

\begin{tabular}{cccccrr}
\hline C.Transp. & C.Est. & ML & OU & LE-NL & LE-NB & LE-D \\
\hline Lt & $\mathrm{Li}$ & $24.4 \%$ & $\mathbf{1 . 6 \%}$ & $7.7 \%$ & $19.4 \%$ & $2.3 \%$ \\
$\mathrm{Lt}$ & $\mathrm{Hi}$ & $\mathbf{1 . 5 \%}$ & $33.5 \%$ & $6.7 \%$ & $4.6 \%$ & $12.8 \%$ \\
$\mathrm{Mt}$ & $\mathrm{Li}$ & $36.5 \%$ & $1.3 \%$ & $4.3 \%$ & $16.3 \%$ & $\mathbf{0 . 6 \%}$ \\
$\mathrm{Mt}$ & $\mathrm{Hi}$ & $14.9 \%$ & $\mathbf{4 . 1 \%}$ & $7.3 \%$ & $13.4 \%$ & $4.8 \%$ \\
$\mathrm{Ht}$ & $\mathrm{Li}$ & $38.4 \%$ & $2.4 \%$ & $2.2 \%$ & $11.6 \%$ & $\mathbf{0 . 5 \%}$ \\
$\mathrm{Ht}$ & $\mathrm{Hi}$ & $24.7 \%$ & $\mathbf{1 . 9 \%}$ & $8.1 \%$ & $20.3 \%$ & $2.2 \%$ \\
\multicolumn{2}{l}{ Desvio Médio Global } & $23.4 \%$ & $7.5 \%$ & $6.0 \%$ & $14.3 \%$ & $\mathbf{3 . 9 \%}$ \\
\hline
\end{tabular}


Custo Médio de Transporte

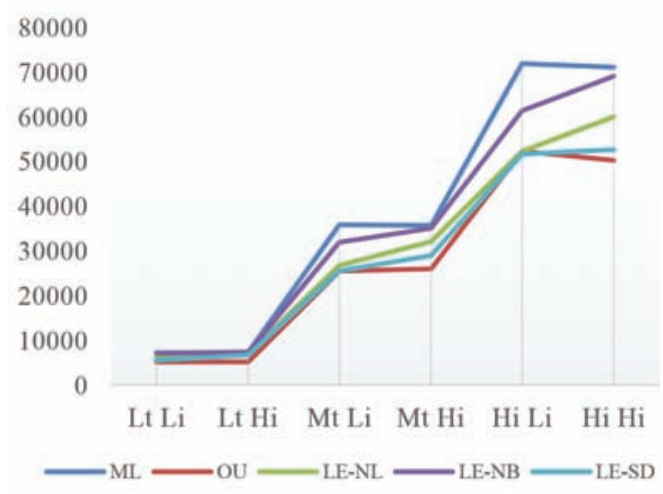

Custo Médio de Estocagem nos

\section{Clientes}

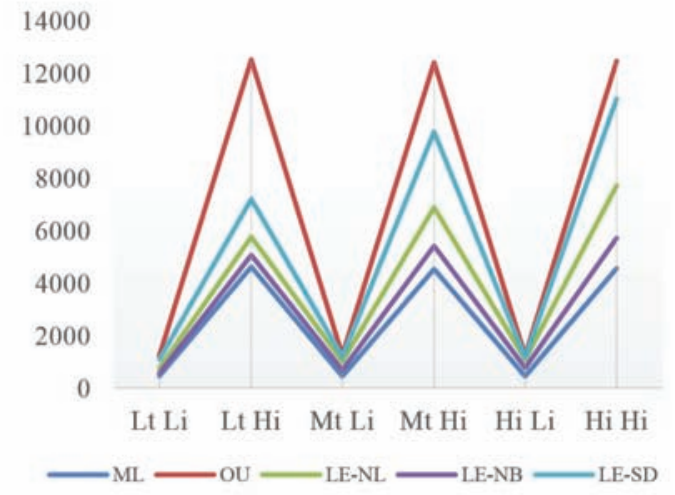

Custo Médio de Estocagem no CD

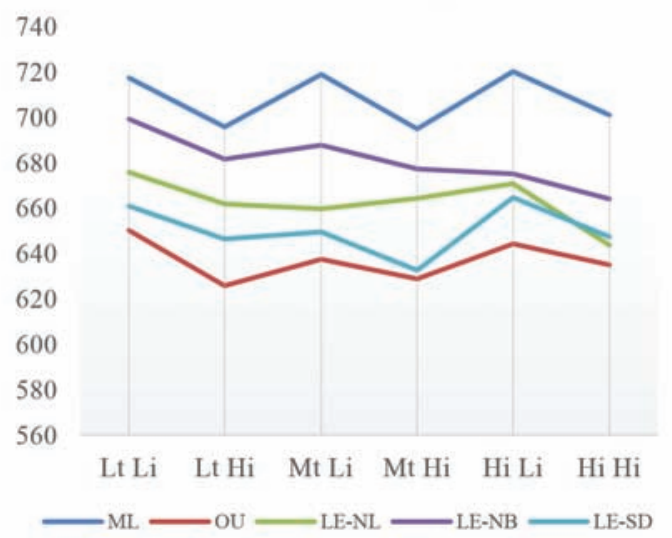

Figura 3. Resumo dos resultados.

ser superior a 63 (número de cenários) devido à possibilidade de empate entre as políticas.

Finalmente, a Tabela 3 mostra os desvios médios para cada uma das políticas em relação ao desvio das melhores soluções médias conhecidas. Com base nesses dados, fica demonstrado que a formulação LE-NL possui desempenho relativo superior à política $O U$, com $6,0 \%$ de desvio médio global em relação à média BKS, contra 7,5\%. Destaca-se o domínio da política LE-D para as configurações Mt-Li e Ht-Li, com desvios inferiores a $1 \%$ para a média BKS. Em termos globais, a política LE-D é superior às demais, seguida da política LE-NL. Demonstra-se, portanto, que a formulação de lote econômico considerando apenas distâncias é mais eficiente que aquelas ponderadas pela quantidade transportada.

\section{Conclusões}

O presente estudo abordou o PRE a partir da óptica da logística de distribuição baseada em sistemas de estoques gerenciados pelo fornecedor em um sistema logístico de três níveis. Foram introduzidas três novas políticas de distribuição baseadas em lote econômico inseridas em uma nova estratégia de atendimento dos clientes, dinamizando o Método de Partição de Conjunto Fixo com a realização a priori da programação das entregas.

Uma nova abordagem heurística foi desenvolvida, dentro da lógica de distribuição por grupos de atendimento. $\mathrm{O}$ algoritmo proposto envolve a resolução do problema em 2 estágios. Depois do processamento das entregas ao longo do horizonte de planejamento, os clientes são agrupados pelo algoritmo k-means modificado para, posteriormente, serem roteirizados por meio de heurísticas clássicas de geração de rotas. Como técnicas de melhoria, as trocas 2-opt foram empregadas e foi proposta uma busca em vizinhança ampla com novos critérios de destruição e reparação da solução incumbente.

Os testes computacionais mostraram-se bastante satisfatórios no que tange ao desempenho das políticas de lote econômico em relação às políticas $M L$ e $O U$. Em geral, os dados médios compartimentados em seis configurações de custos 
distintas demonstraram um predomínio da política de lote econômico baseado somente em distâncias em relação às outras políticas. Dentre uma gama de 378 cenários analisados com número de clientes variando de 5 a 100, número de períodos com 5,10 e 20 e número de veículos entre 2,3 e 4 , a política LE-D foi vitoriosa em aproximadamente $40 \%$ das instâncias.

Analisando a performance de cada política com relação às diferentes combinações de custos, a política $M L$ é endereçada para problemas em que os clientes são localizados próximos uns dos outros e o custo de estocagem do produto é elevado. Já a política $O U$ consegue ser superior para problemas com elevado custo de estocagem desde que os clientes possuam média ou elevada dispersão locacional (médio ou alto custo de transporte). Problemas com baixo custo de estocagem também são indicados à política $O U$ desde que o custo de estocagem seja baixo. A LE-D é mais eficiente em problemas intermediários, com médio custo de transporte e baixo custo de estocagem além da configuração com alto custo de transporte e baixo custo de estocagem. Embora a formulação LE-NL não tenha produzido menores desvios de forma segmentada por configuração de custos, seu desempenho é superior em termos globais em relação à política $O U$. Observa-se ainda que a política de lote econômico baseada nas necessidades brutas possui resultados melhores em termos globais apenas em comparação à política $M L$.

O desempenho da heurística no tocante ao tempo de processamento foi bastante satisfatório e estável, demandando em média 100 segundos independente da política de distribuição ou da configuração de custo do cenário analisado.

\section{Referências}

ADULYASAK, Y.; CORDEAU, J.; JANS, R. Optimizationbased adaptive large neighborhood search for the production routing problem. Transportation Science, v. 48, n. 1, p. 20-45, 2012. Disponível em: <http://transci.journal.informs.org/cgi/doi/10.1287/ trsc.1120.0443>.

ALEGRE, J.; LAGUNA, M.; PACHECO, J. Optimizing the periodic pick-up of raw materials for a manufacturer of auto parts. European Journal of Operational Research, v. 179, n. 3, p. 736-746, 2007. Disponível em: <http://linkinghub.elsevier.com/retrieve/pii/ S0377221705007332>.

ANILY, S.; BRAMEL, J. An asymptotic 98.5\%-effective lower bound on fixed partition policies for the inventory-routing problem. Discrete Applied Mathematics, v. 145, n. 1, p. 22-39, 2004. Disponível em: <http://linkinghub.elsevier.com/retrieve/pii/ S0166218X04000630>.

ARCHETTI, C. et al. A branch-and-cut algorithm for a vendor-managed inventory-routing problem. Transportation Science, v. 41, n. 3, p. 382-391, 2007.
Disponível em: <http://transci.journal.informs.org/cgi/ doi/10.1287/trsc.1060.0188>.

ARCHETTI, C. et al. A hybrid heuristic for an inventory routing problem. INFORMS Journal on Computing, v. 24, n. 1, p. 101-116, 2011. Disponível em: <http://joc. journal.informs.org/cgi/doi/10.1287/ijoc.1100.0439>.

BERTAZZI, L.; PALETTA, G.; SPERANZA, M. Minimizing the total cost in an integrated vendor managed inventory system. Journal of Heuristics, v. 11, n. 5-6, p. 393-419, 2005. Disponível em: <http://www.springerlink.com/index/10.1007/ s10732-005-0616-6>.

BIRGER, R.; EL-HOUSSAINE, A. Designing distribution patterns for long-term inventory routing with constant demand rates. International Journal of Production Economics, v. 112, n. 1, p. 255-263, 2008. Disponível em: <http://linkinghub.elsevier.com/retrieve/pii/ S0925527307001442>.

BOUDIA, M.; PRINS, C. A memetic algorithm with dynamic population management for an integrated production-distribution problem. European Journal of Operational Research, v. 195, n. 3, p. 703-715, 2009. Disponível em: <http://linkinghub.elsevier.com/ retrieve/pii/S037722170701096X>.

CHAVES, A. A. Uma metaheurística híbrida com busca por agrupamentos aplicada à problemas de otimização combinatoria. 2009. 197 f. Tese (Doutorado em Computação Aplicada)-Instituto Nacional de Pesquisas Espaciais, São José dos Campos, 2009.

CHRISTIANSEN, M. et al. Maritime inventory routing with multiple products: a case study from the cement industry. European Journal of Operational Research, v. 208, n. 1, p. 86-94, 2011. Disponível em: <http://linkinghub.elsevier.com/retrieve/pii/ S0377221710005606>.

COELHO, L. C.; CORDEAU, J.; LAPORTE, G. Thirty years of inventory routing. Transportation Science, v. 48 , n. 1, p. 1-19, 2013. http://dx.doi.org/10.1287/ trsc.2013.0472

COELHO, L. C.; LAPORTE, G. An optimized target level inventory replenishment policy for vendormanaged inventory systems. Montreal: CIRRELT, 2013.

COELHO, L. Flexibility and consistency in inventory-routing. 2012. $162 \mathrm{f}$. Tese (Doutorado em Administração)-HEC Montréal, Université de Montréal, Montréal, 2012. Disponível em: <http:// www.hec.ca/programmes_formations/phd/theses/2011/ resume/prop-CALLEGARI COELHO.pdf>.

COELHO, L.; CORDEAU, J.; LAPORTE, G. Consistency in multi-vehicle inventory-routing. Transportation Research Part C: Emerging Technologies, v. 24, p. 270-287, 2012a. Disponível em: <http://linkinghub. elsevier.com/retrieve/pii/S0968090X12000502>.

COELHO, L.; CORDEAU, J.; LAPORTE, G. The inventory-routing problem with transshipment. Computers \& Operations Research, v. 39, n. 11, p. 2537-2548, 2012b. Disponível em: <http://linkinghub. elsevier.com/retrieve/pii/S0305054812000032>. 
COELHO, L.; LAPORTE, G.; CORDEAU, J. Dynamic and stochastic inventory-routing. Montreal: CIRRELT, 2012c. (Technical Report CIRRELT2012-37). Disponível em: <https://www.cirrelt.ca/ DocumentsTravail/CIRRELT-2012-37.pdf $>$.

DÍAZ, J. A.; FERNÁNDEZ, E. Hybrid scatter search and path relinking for the capacitated p-median problem. European Journal of Operational Research, v. 169, n. 2, p. 570-585, 2006. Disponível em: <http://linkinghub.elsevier.com/retrieve/pii/ S0377221704005557>.

GEETHA, S.; POONTHALIR, G.; VANATHI, P. Improved $\mathrm{k}$-means algorithm for capacitated clustering problem. INFOCOMP Journal of Computer Science, v. 8, n. 4, p. 52-59, 2009. Disponível em: <http://www.dcc.ufla. br/infocomp/artigos/v8.4/art07.pdf $>$.

GOEL, V. et al. Large neighborhood search for LNG inventory routing. Journal of Heuristics, v. 18, n. 6 , p. 821-848, 2012. Disponível em: <http://link.springer. com/10.1007/s10732-012-9206-6>

GRONHAUG, R. et al. A branch-and-price method for a liquefied natural gas inventory routing problem. Transportation Science, v. 44, n. 3, p. 400-415, 2010. Disponível em: <http://transci.journal.informs.org/cgi/ doi/10.1287/trsc.1100.0317>.

GUMASTA, K.; CHAN, F. T. S.; TIWARI, M. K. An incorporated inventory transport system with two types of customers for multiple perishable goods. International Journal of Production Economics, v. 139, n. 2, p. 678-686, 2012. Disponível em: <http://linkinghub.elsevier.com/retrieve/pii/ S0925527312002654>.

HEMMELMAYR, V. et al. Vendor managed inventory for environments with stochastic product usage. European Journal of Operational Research, v. 202, n. 3, p. 686-695, 2010. Disponível em: <http://linkinghub. elsevier.com/retrieve/pii/S037722170900424X>.

HIASSAT, A. H.; DIABAT, A. A location inventory routing problem with perishable products. In: INTERNATIONAL CONFERENCE ON COMPUTERS \& INDUSTRIAL ENGINEERING, 41., 2010, Los Angeles. Proceedings... p. 386-391.

KAVEH, P.; ZADEH, A. S.; SAHRAEIAN, R. Solving capacitated p-median problem by hybrid k-means clustering and FNS algorithm. International Journal of Innovation, Management and Technology, v. 1, n. 4, p. 405-410, 2010.

LI, J.; CHEN, H.; CHU, F. Performance evaluation of distribution strategies for the inventory routing problem. European Journal of Operational Research, v. 202, n. 2, p. 412-419, 2010. Disponível em: <http://linkinghub.elsevier.com/retrieve/pii/ S0377221709003506>.

LIAO, K.; GUO, D. A clustering-based approach to a special capacitated facility location problem. Transactions in GIS, v. 12, n. 3, p. 323-339, 2008. http://dx.doi.org/10.1111/j.1467-9671.2008.01105.x

LIN, S.; KERNIGHAN, B. W. An effective heuristic algorithm for the traveling-salesman problem. Operations Research, v. 21, n. 2, p. 498-516, 1973. http://dx.doi.org/10.1287/opre.21.2.498
LIU, S.-C.; CHEN, A.-Z. Variable neighborhood search for the inventory routing and scheduling problem in a supply chain. Expert Systems with Applications, v. 39, n. 4, p. 4149-4159, 2012. Disponível em: <http://linkinghub. elsevier.com/retrieve/pii/S0957417411014436>.

LIU, S.-C.; LEE, W.-T. A heuristic method for the inventory routing problem with time windows. Expert Systems with Applications, v. 38, n. 10, p. 1322313231, 2011. Disponível em: <http://linkinghub. elsevier.com/retrieve/pii/S0957417411006658>.

NANANUKUL, N. Clustering model and algorithm for production inventory and distribution problem. Applied Mathematical Modelling, v. 37, n. 24, p. 9846-9857, 2013. Disponível em: <http://linkinghub.elsevier.com/ retrieve/pii/S0307904X13003508>.

NISHI, T.; IZUNO, T. Column generation heuristics for ship routing and scheduling problems in crude oil transportation with split deliveries. Computers \& Chemical Engineering, v. 60, p. 329-338, 2014. Disponível em: <http://linkinghub.elsevier.com/ retrieve/pii/S0098135413003001>.

ÖZENER, O. Ö.; ERGUN, Ö.; SAVELSBERGH, M. Allocating cost of service to customers in inventory routing. Operations Research, v. 61, n. 1, p. 112-125, 2013. http://dx.doi.org/10.1287/opre.1120.1130

PISINGER, D.; ROPKE, S. Large neighborhood search. In: GENDREAU, M.; POTVIN, J.-Y. (Ed.). Handbook of metaheuristics. New York: Springer, 2010. p. 1-22. Disponível em: <http://link.springer.com/ chapter/10.1007/978-1-4419-1665-5_13>.

POP, P. C.; KARA, I.; MARC, A. H. New mathematical models of the generalized vehicle routing problem and extensions. Applied Mathematical Modelling, v. 36, n. 1, p. 97-107, 2012. Disponível em: <http://linkinghub. elsevier.com/retrieve/pii/S0307904X11003489>.

POPOVIĆ, D.; VIDOVIĆ, M.; RADIVOJEVIĆ, G. Variable neighborhood search heuristic for the inventory routing problem in fuel delivery. Expert Systems with Applications, v. 39, n. 18, p. 1339013398, 2012. Disponível em: <http://linkinghub. elsevier.com/retrieve/pii/S0957417412007804>.

RAA, B.; AGHEZZAF, E.-H. A practical solution approach for the cyclic inventory routing problem. European Journal of Operational Research, v. 192, n. 2, p. 429-441, 2009. Disponível em: <http://linkinghub. elsevier.com/retrieve/pii/S0377221707009526>.

RAKKE, J. G. et al. A rolling horizon heuristic for creating a liquefied natural gas annual delivery program. Transportation Research Part C: Emerging Technologies, v. 19, n. 5, p. 896-911, 2011. Disponível em: <http://linkinghub.elsevier.com/retrieve/pii/ S0968090X10001427>

ROSENKRANTZ, D. J.; STEARNS, R. E.; LEWIS, P. M. An analysis of several heuristics for the traveling salesman problem. SIAM Journal on Computing, v. 6, n. 3, p. 563-581, 1977. http://dx.doi. org/10.1137/0206041

SCHMID, V.; DOERNER, K. F.; LAPORTE, G. Rich routing problems arising in supply chain management. European Journal of Operational Research, v. 224, 
n. 3, p. 435-448, 2013. Disponível em: <http://dx.doi. org/10.1016/j.ejor.2012.08.014>.

SCHWARZ, L. B. Flexibility principles. In: CHHAJED, D.; LOWE, T. J. (Ed.). Building intuition: insights from basic operations management models and principles. Boston: Springer, 2008. Disponível em: <http://www. springerlink.com/index/10.1007/978-0-387-73699-0>.

SHAW, P. Using constraint programming and local search methods to solve ve- hicle routing problems. In: INTERNATIONAL CONFERENCE ON PRINCIPLES AND PRACTICE OF CONSTRAINT PROGRAMMING (CP-98), 4., 1998, Pisa, Italy. Springer Berlin Heidelberg, 1998. p. 417-431. (Lecture Notes in Computer Science, 1520).

SHUKLA, N. et al. Algorithm portfolios for logistics optimization considering stochastic demands and mobility allowance. International Journal of Production Economics, v. 141, n. 1, p. 146-166, 2013. Disponível em: <http://linkinghub.elsevier.com/ retrieve/pii/S0925527312003143>.

SINDHUCHAO, S. et al. An integrated inventoryrouting system for multi-item joint replenishment with limited vehicle capacity. Journal of Global Optimization, v. 32, n. 1, p. 93-118, 2005. Disponível em: <http://www.springerlink.com/index/10.1007/ s10898-004-5908-0>.

SOLYALI, O.; SURAL, H. A branch-and-cut algorithm using a strong formulation and an a priori tourbased heuristic for an inventory-routing problem.

Transportation Science, v. 45, n. 3, p. 335-345, 2011. Disponível em: <http://transci.journal.informs.org/cgi/ doi/10.1287/trsc.1100.0354>.

WANKE, P. Quadro conceitual para gestão de estoques : enfoque nos itens. Gestão \& Produção, v. 19, n. 4, p. 677-687, 2012a. http://dx.doi.org/10.1590/ S0104-530X2012000400002

WANKE, P. Product, operation, and demand relationships between manufacturers and retailers. Transportation Research Part E: Logistics and Transportation Review, v. 48, n. 1, p. 340-354, 2012b. Disponível em: <http://linkinghub.elsevier.com/retrieve/pii/ S1366554511001050>.

ZHAO, Q.-H.; CHEN, S.; ZANG, C.-X. Model and algorithm for inventory/routing decision in a threeechelon logistics system. European Journal of Operational Research, v. 191, n. 3, p. 623-635, 2008. Disponível em: <http://linkinghub.elsevier.com/ retrieve/pii/S037722170700121X>. 